\title{
Previsão de Curvas de Titulação (II): Ácidos Polipróticos
}

\author{
J.L.G.F.S. Costa Pereira *
}

\begin{abstract}
$R$ ecorrendo a um método numérico previamente descrito procurou-se neste trabalho estender a previsão das curvas de titulação ao caso poliprótico. Foram aqui deduzidas algumas expressões que permitem construir, para cada caso, o modelo matemático correcto, isento das tradicionais aproximações e simplificações. Após o estudo de três casos concretos de titulação de um ácido diprótico $\left(\mathrm{H}_{2} \mathrm{~A}\right)$, triprótico $\left(\mathrm{H}_{3} \mathrm{~A}\right)$ e tetraprótico $\left(\mathrm{H}_{4} \mathrm{~A}\right)$ com uma base forte $(\mathrm{MOH})$, foi deduzida uma expressão genérica válida para todas as situações de ácido poliprótico $\left(\mathrm{H}_{\mathrm{n}} \mathrm{A}\right)$. Verificou-se ainda que o modelo desenvolvido consegue descrever todas os tipos de titulação de ácidos com base forte, inclusive os casos em que se trata da titulação de um ácido forte $(\mathrm{HX})$ e de sistemas que envolvem equilíbrios simultâneos, como no caso de ácidos polipróticos com constantes de equilíbrio com valores muito próximos. Este tipo de abordagem matemático-numérica apresenta ainda como vantagens não só a possibilidade de permitir a estimativa das respectivas constantes de equilíbrio como ainda a de facultar a possibilidade de simulação e previsão do comportamento químico das espécies em solução aquosa e, desse modo, facultar ilações sobre o sistema em estudo.
\end{abstract}

Palavras chave

curva de titulação potenciométrica, modelo, previsão, reacções ácido-base, ácidos polipróticos

\section{INTRODUÇÃO}

A reactividade do tipo ácido-base parece ser um conceito químico simples e fácil de interpretar, razão pela qual, este é um dos primeiros assuntos a ser apresentado aos alunos ao nível da aprendizagem da estequiometria, dinâmica de reactividade e equilíbrio químico [1, 2]. Contudo, nota-se que este assunto não está totalmente esclarecido e deve ser alvo de uma análise mais atenta e detalhada.

Este tipo de reacções químicas é de facto muito comum sendo mais frequente e evidente nas situações em que se processa a transferência de protão $\left(\mathrm{H}^{+}\right)$, tal como anteriormente descrito em 1923 por Brønsted e Lowry'.

A relevância deste simples fenómeno, transferência de protão, está muito re-

Departamento de Química da Faculdade de Ciências e Tecnologia da Universidade de Coimbra

3004-535 Coimbra

e-mail: jcpereira@qui.uc.p lacionada com as propriedades únicas que a água desempenha enquanto solvente. Sendo a água relativamente abundante, ao nível deste planeta, e estando esta presente numa infinidade de situações, compreende-se a imensidão de fenómenos do tipo ácido-base. Hoje em dia sabe-se inclusivamente que, em algumas outras situações mais drásticas, a transferência de protão pode processar-se directamente sem a participação da água, como intermediário, alargando ainda mais o domínio da possibilidade de ocorrência destas reacções.

O predomínio das reacções do tipo ácido-base em toda a reactividade química está ainda relacionado com o facto de diversos compostos químicos apresentarem uma relativa facilidade para participarem em fenómenos de transferência de protão, razão pela qual é possível identificar a presença de reacções ácido-base numa infinidade de sistemas químicos e bioquímicos [3].
Pensa-se inclusivamente que, devido às características anfifílicas ${ }^{2}$ que diversos compostos biológicos possuem, a transferência de protão ao nível das biomembranas lipídicas, promovida por uma série de espécies químicas situadas na sua interface ou ainda embutidas nas próprias biomembranas, possa estar na génese da existência da própria vida, tal como a conhecemos [4].

As reacções do tipo ácido-base são também muito relevantes nos dias de hoje já que estão na origem de diversos fenómenos relacionados com aplicações industriais quer ao nível da indústria química tradicional, quer ao nível das indústrias alimentar, farmacêutica, etc.

Embora a relevância deste tipo de fenómeno químico tenha todo este impacto científico-tecnológico, o presente trabalho visa, sob uma perspectiva analítica, procurar descrever e prever o comportamento de qualquer ácido 
em meio aquoso permitindo antever, por simulação, particularidades desse sistema.

De acordo com as bases de dados bibliográficas Science Direct e ISI Web of Knowledge, a simulação de curvas de titulação começou em 1978 com a publicação do trabalho de James, Davis e Leckie denominado "Computer simulation of the conductometric and potentiometric titrations of the surface groups on ionizable latexes" [5].

Desde então diversos autores têm investido no sentido de melhor compreender e descrever este fenómeno em diversas situações distintas, havendo inclusivamente diversas empresas que disponibilizam versões de software para o efeito.

Referimos ainda que, na Internet, existe inclusivamente uma folha de Excel, desenvolvida e mantida por Ivano Gebhardt Rolf Gutz desde 1992, denominada de "CurTiPot" que se encontra disponibilizada através do endereço http://www2.iq.usp.br/docente/gutz/ Curtipot.html.

Contudo, a nossa postura mais educativa e formativa vai no sentido de explicar a fundamentação físico-química subjacente e procurar incentivar o leitor no sentido de desenvolver a sua própria ferramenta de trabalho.

Convém ainda salientar que o procedimento anteriormente descrito [6], aqui utilizado e desenvolvido, permite descrever todo o sistema químico, presente na solução do titulado, sem ter que se recorrer a grosseiras aproximações teóricas ou segmentações do comportamento químico do sistema.

Este facto está relacionado com o facto do tipo de tratamento matemáticonumérico sugerido facultar o contorno da dificuldade analítica imposta pela resolução de equações de grau mais elevado.

Esta abordagem integral do sistema químico como um todo fundamentase essencialmente em dois princípios aplicáveis às soluções de electrólito em sistema fechado: balanços de massa e de carga das espécies presentes em solução.
Após a adaptação do balanço mássico para estar em conformidade com as condições de sistema aberto de uma titulação, os modelos desenvolvidos servem para descrever adequadamente os resultados obtidos em meio aquoso, por via potenciométrica, de qualquer espécie ácida $\left(\mathrm{H}_{\mathrm{n}} \mathrm{A}\right) \mathrm{com}$ uma base forte $(\mathrm{MOH})$.

\section{APROXIMAÇões E FUNDAMENTAÇÃo}

Este trabalho foi concebido no sentido de minimizar os erros de carbonatação das soluções aquosas durante a titulação ácido-base, razão pela qual, o ácido será sempre colocado no recipiente do titulado enquanto que na bureta se encontra a solução da base [6].

Também exclusivamente por razões analíticas e para simplificar esta abordagem o titulante utilizado é sempre uma monobase ${ }^{3}$ forte, aqui representada de $\mathrm{MOH}$. São exemplos desta base os hidróxidos de metais alcalinos ( $\mathrm{LiOH}, \mathrm{NaOH}, \mathrm{KOH})$.

Para os sistemas em análise assumese que apenas existem reacções rápidas de transferência de protão caracterizadas pelas respectivas constantes de autoprotólise da água $\left(\mathrm{K}_{\mathrm{w}}\right) \mathrm{e}$ ionização dos ácidos $\left(\mathrm{K}_{\mathrm{i}}\right)$.

Deste modo, em qualquer instante da curva de titulação, a concentração das diversas espécies presentes na solução do titulado é condicionada apenas pelas respectivas constantes de equilíbrio.

Apenas por conveniência numérica, para simplificar este tratamento matemático-numérico, assume-se que, durante a titulação, estas constantes de equilíbrio não variam ${ }^{4}$.

Sendo este trabalho do foro analítico, o valor das constantes a utilizar na previsão da respectiva curva de titulação refere-se ao produto e/ou quociente de concentrações de equilí-

$$
\begin{gathered}
\alpha_{0}=\frac{\left[\mathrm{H}_{n} \mathrm{~A}\right]}{C_{a}}=\frac{\left[\mathrm{H}^{+}\right]^{n}}{\sum_{i=0}^{n}\left[\left(\prod_{j=1}^{i} \mathrm{~K}_{j}\right) \cdot\left[\mathrm{H}^{+}\right]^{(n-i)}\right]} \\
\alpha_{1}=\frac{\left[\mathrm{H}_{(n-1)} \mathrm{A}^{-}\right]}{C_{a}}=\frac{\mathrm{K}_{1}\left[\mathrm{H}^{+}\right]^{(n-1)}}{\sum_{i=0}^{n}\left[\left(\prod_{j=1}^{i} \mathrm{~K}_{j}\right) \cdot\left[\mathrm{H}^{+}\right]^{(n-i)}\right]}
\end{gathered}
$$

brio (constantes condicionais) embora possam ser utilizadas as respectivas estimativas termodinâmicas, válido para o caso de soluções muito diluídas. Com base nesta aproximação, o produto iónico da água é dado por:

$\mathrm{K}_{w}=\left[\mathrm{H}^{+}\right] \cdot\left[\mathrm{OH}^{-}\right]$

Um ácido poliprótico $\left(\mathrm{H}_{\mathrm{n}} \mathrm{A}, \mathrm{n}=\{2,3\right.$, ...\}) em meio aquoso ioniza-se sucessivamente através de $n$ equilíbrios químicos até atingir a sua forma totalmente desprotonada $\left(\mathrm{A}^{\mathrm{n}-}\right)$.

As respectivas constantes de equilíbrio em cada etapa de ionização $\left(\mathrm{K}_{\mathrm{i}}\right.$, $i=\{1,2, \ldots, n\})$ são dadas pelos seguintes quocientes de concentração:

$$
\begin{aligned}
& \mathrm{K}_{1}=\frac{\left[\mathrm{H}^{+}\right] \cdot\left[\mathrm{H}_{(n-1)} \mathrm{A}^{-}\right]}{\left[\mathrm{H}_{n} \mathrm{~A}\right]} \\
& \mathrm{K}_{2}=\frac{\left[\mathrm{H}^{+}\right] \cdot\left[\mathrm{H}_{(n-2)} \mathrm{A}^{2-}\right]}{\left[\mathrm{H}_{(n-1)} \mathrm{A}^{-}\right]} \ldots \\
& \mathrm{K}_{i}=\frac{\left[\mathrm{H}^{+}\right] \cdot\left[\mathrm{H}_{(n-i)} \mathrm{A}^{i-}\right]}{\left[\mathrm{H}_{(n-i+1)} \mathrm{A}^{(i-1)-}\right]} \\
& \ldots \\
& \mathrm{K}_{n}=\frac{\left[\mathrm{H}^{+}\right] \cdot\left[\mathrm{A}^{n-}\right]}{\left[\mathrm{HA}^{(n-1)-}\right]}
\end{aligned}
$$

No sistema considerado, o número de moles de ácido presentes na solução mantém-se constante sendo o respectivo balanço de massa num determinado instante $i$ da titulação traduzido através da equação:

$$
\begin{aligned}
& C_{a}(i)=\left[\mathrm{H}_{n} \mathrm{~A}\right]+\left[\mathrm{H}_{(n-1)} \mathrm{A}^{-}\right]+ \\
& +\left[\mathrm{H}_{(n-2)} \mathrm{A}^{2-}\right]+\ldots+\left[\mathrm{HA}^{(n-1)-}\right]+\left[\mathrm{A}^{n-}\right]
\end{aligned}
$$

Por substituição das equações (0.2) em (0.3) podem ser deduzidas as expressões para as respectivas fracções molares de cada uma das formas do ácido presente na solução do titulado: 


$$
\begin{gathered}
\alpha_{i}=\frac{\left[\mathrm{H}_{(n-i)} \mathrm{A}^{i-}\right]}{C_{a}}=\frac{\left(\prod_{j=1}^{i} \mathrm{~K}_{j}\right) \cdot\left[\mathrm{H}^{+}\right]^{(n-i)}}{\sum_{i=0}^{n}\left[\left(\prod_{j=1}^{i} \mathrm{~K}_{j}\right) \cdot\left[\mathrm{H}^{+}\right]^{(n-i)}\right]} \\
\alpha_{n}=\frac{\left[\mathrm{A}^{n-}\right]}{C_{a}}=\frac{\prod_{j=1}^{n} \mathrm{~K}_{j}}{\sum_{i=0}^{n}\left[\left(\prod_{j=1}^{i} \mathrm{~K}_{j}\right) \cdot\left[\mathrm{H}^{+}\right]^{(n-i)}\right]}
\end{gathered}
$$

Em qualquer instante da titulação o valor de $\mathrm{pH}$ da solução pode ser estimado com base na concentração analítica do hidrogenião $\left(\mathrm{H}^{+}\right)$:

$$
\mathrm{pH}=-\log a_{\mathrm{H}^{+}} \simeq-\log \left[\mathrm{H}^{+}\right]
$$

Por razões de simplificação analítica, como titulante utiliza-se apenas uma monobase forte $(\mathrm{MOH})$, de concentração $C_{b}$, de forma a que, em solução aquosa diluída $(\mathrm{C}<1 \mathrm{M})$, este electrólito forte se encontra totalmente ionizado, permanecendo o seus iões $\left(\mathrm{M}^{+}\right.$e $\left.\mathrm{OH}^{-}\right)$sob a forma hidratada. Deste modo o catião do titulante $\left(\mathrm{M}^{+}\right)$ não interfere no processo ácido-base que decorre na solução titulada.

Apenas por uma questão de simplificação das expressões define-se o volume equivalente $\left(\mathrm{V}_{\text {eq }}\right)$ como sendo a quantidade de titulante necessária para neutralizar uma mole equivalente do ácido poliprótico $\left(\mathrm{C}_{\mathrm{a}} \cdot \mathrm{V}_{\mathrm{a}}\right)$ com uma mole de base monoprótica $\left(C_{b} \cdot V_{e q}\right)$.

$$
V_{e q}=V_{a} \frac{C_{a}}{C_{b}}
$$

Deste modo um ácido poliprótico com $n$ protões $\left(\mathrm{H}_{\mathrm{n}} \mathrm{A}\right)$ é completamente titulado com um volume de base correspondente $\mathrm{a}\left(\mathrm{n} \times \mathrm{V}_{\mathrm{eq}}\right)$.

A titulação é seguida através do registo do valor do $\mathrm{pH}$ da solução titulada em função do volume de base adicionada $\left(\mathrm{V}_{\mathrm{b}}\right)$.

Sendo a solução do titulado um sistema aberto, todas a concentrações nesta solução devem ser recalculadas em cada instante através do respectivo factor de diluição. No instante $i$ da titulação, a concentração total de ácido no titulado, $\mathrm{C}_{\mathrm{a}}(i)$, é dada por:

$$
C_{a}(i)=C_{a} \cdot \frac{V_{a}}{V_{a}+V_{b}}
$$

Estas aproximações e pressupostos permitem deduzir as seguintes equações para a previsão e modelação de curvas de titulação, dependendo do tipo de estimativa a efectuar.

Neste trabalho são analisados quatro casos relacionados com a titulação de espécies ácidas com uma base forte (MOH): a) titulação de um ácido diprótico $\left.\left(\mathrm{H}_{2} \mathrm{~A}\right), b\right)$ titulação de um ácido triprótico $\left.\left(\mathrm{H}_{3} \mathrm{~A}\right), c\right)$ titulação de um ácido tetraprótico $\left(\mathrm{H}_{4} \mathrm{~A}\right)$ e d) titulação de um ácido poliprótico $\left(\mathrm{H}_{\mathrm{n}} \mathrm{A}\right)$.

C UASO 1 - TITULAÇÃO DE ÁCIDO DIPRÓTICO $\left(\mathrm{H}_{2} \mathrm{~A}\right)$ COM UMA BASE FORTE (MOH)

A titulação processa-se em duas etapas:

$$
\begin{aligned}
& \mathrm{H}_{2} \mathrm{~A}(a q)+\mathrm{OH}^{-}(a q) \rightarrow \mathrm{HA}^{-}(a q)+\mathrm{H}_{2} \mathrm{O}(l) \\
& \mathrm{HA}^{-}(a q)+\mathrm{OH}^{-}(a q) \rightarrow \mathrm{A}^{2-}(a q)+\mathrm{H}_{2} \mathrm{O}(l)
\end{aligned}
$$

que podem ser sequenciais ou quase simultâneas, dependendo da proximidade relativa das constantes de ionização sucessiva.

Regra geral, considera-se que quando as constantes sucessivas diferem mais de três ordens de grandeza os equilíbrios são independentes e a titulação decorre em etapas individualizadas, cada uma caracterizada pelo seu ponto de equivalência.

Inicialmente é colocado no vaso de titulação o volume $V_{a}$ de ácido com concentração $C_{a}$. Após a adição do volume $V_{b}$ de titulante de concentração $C_{b}$, o princípio da electroneutralidade das soluções de electrólito assume a existência do seguinte equilíbrio iónico:

$$
\left[\mathrm{H}^{+}\right]+\left[\mathrm{M}^{+}\right]=\left[\mathrm{OH}^{-}\right]+\left[\mathrm{HA}^{-}\right]+2\left[\mathrm{~A}^{2-}\right]
$$

As concentrações anteriores podem ser estimadas através de:

$$
\begin{gathered}
{\left[\mathrm{M}^{+}\right]=\frac{C_{b} \cdot V_{b}}{V_{a}+V_{b}}} \\
{\left[\mathrm{OH}^{-}\right]=\frac{\mathrm{K}_{w}}{\left[\mathrm{H}^{+}\right]}} \\
{\left[\mathrm{HA}^{-}\right]+2\left[\mathrm{~A}^{2-}\right]=\frac{C_{b} \cdot V_{e q}}{V_{a}+V_{b}} \cdot\left(\alpha_{1}+2 \alpha_{2}\right)}
\end{gathered}
$$

onde $\left(\alpha_{1}\right.$ e $\left.\alpha_{2}\right)$ são as fracções molares das formas ionizadas do ácido, equações (0.4).

Por substituição em (1.1) obtém-se a expressão simplificada:

$$
\begin{aligned}
& {\left[\mathrm{H}^{+}\right]-\frac{K_{w}}{\left[\mathrm{H}^{+}\right]}+\frac{C_{b}}{V_{a}+V_{b}} .} \\
& .\left(V_{b}-\alpha_{a 2}^{*} \cdot V_{e q}\right)=0
\end{aligned}
$$

onde

$$
\begin{aligned}
& \alpha_{a 2}^{*}=\alpha_{1}+2 \alpha_{2}= \\
& =\frac{\mathrm{K}_{1}\left[\mathrm{H}^{+}\right]+2 \mathrm{~K}_{1} \mathrm{~K}_{2}}{\left[\mathrm{H}^{+}\right]^{2}+\mathrm{K}_{1}\left[\mathrm{H}^{+}\right]+\mathrm{K}_{1} \mathrm{~K}_{2}}
\end{aligned}
$$

representa a fracção molar equivalente de ácido diprótico (a2) que se encontra em solução sob a forma ionizada.

A equação (1.3) é uma função de terceiro grau em relação à concentração hidrogeniónica não sendo trivial nem recomendável a sua resolução por via analítica.

\section{Caso 2 - Titulação de ácido triprótico $\left(\mathrm{H}_{3} \mathrm{~A}\right)$ COM UMA BASE FORTE (MOH)}

Neste caso a titulação processa-se em três passos:

$\mathrm{H}_{3} \mathrm{~A}(a q)+\mathrm{OH}^{-}(a q) \rightarrow \mathrm{H}_{2} \mathrm{~A}^{-}(a q)+\mathrm{H}_{2} \mathrm{O}(l)$
$\mathrm{H}_{2} \mathrm{~A}^{-}(a q)+\mathrm{OH}^{-}(a q) \rightarrow \mathrm{HA}^{2-}(a q)+\mathrm{H}_{2} \mathrm{O}(l)$
$\mathrm{HA}^{2-}(a q)+\mathrm{OH}^{-}(a q) \rightarrow \mathrm{A}^{3-}(a q)+\mathrm{H}_{2} \mathrm{O}(l)$

sequenciais ou simultâneos dependendo do valor das respectivas constantes de ionização do ácido. 
Em qualquer instante da titulação o equilíbrio iónico estabelecido na solução titulada é traduzido pela seguinte equação:

$$
\begin{aligned}
& {\left[\mathrm{H}^{+}\right]+\left[\mathrm{M}^{+}\right]=\left[\mathrm{OH}^{-}\right]+\left[\mathrm{H}_{2} \mathrm{~A}^{-}\right]+} \\
& +2\left[\mathrm{HA}^{2-}\right]+3\left[\mathrm{~A}^{3-}\right]
\end{aligned}
$$

sendo as concentrações do ácido na forma ionizada dadas por:

$$
\begin{aligned}
& {\left[\mathrm{H}_{2} \mathrm{~A}^{-}\right]+2\left[\mathrm{HA}^{2-}\right]+3\left[\mathrm{~A}^{3-}\right]=} \\
& =\frac{C_{b} \cdot V_{e q}}{V_{a}+V_{b}} \cdot\left(\alpha_{1}+2 \alpha_{2}+3 \alpha_{3}\right)
\end{aligned}
$$

Por substituição em (2.1) e após alguma simplificação dessa expressão chega-se a:

$$
\begin{aligned}
& {\left[\mathrm{H}^{+}\right]-\frac{K_{w}}{\left[\mathrm{H}^{+}\right]}+\frac{C_{b}}{V_{a}+V_{b}} .} \\
& .\left(V_{b}-\alpha_{a 3}^{*} \cdot V_{e q}\right)=0
\end{aligned}
$$

onde

$\alpha_{a 3}^{*}=\alpha_{1}+2 \alpha_{2}+3 \alpha_{3}=$

$\frac{\mathrm{K}_{1}\left[\mathrm{H}^{+}\right]^{2}+2 \mathrm{~K}_{1} \mathrm{~K}_{2}\left[\mathrm{H}^{+}\right]+3 \mathrm{~K}_{1} \mathrm{~K}_{2} \mathrm{~K}_{3}}{\left[\mathrm{H}^{+}\right]^{3}+\mathrm{K}_{1}\left[\mathrm{H}^{+}\right]^{2}+\mathrm{K}_{1} \mathrm{~K}_{2}\left[\mathrm{H}^{+}\right]+\mathrm{K}_{1} \mathrm{~K}_{2} \mathrm{~K}_{3}}$

representa a fracção molar equivalente de ácido triprótico (a3) sob a forma ionizada.

Neste caso, a equação (2.3) é agora uma função de quarto grau em relação à concentração hidrogeniónica não podendo ser por isso resolvida analiticamente pelos métodos correntes.

\section{Caso 3 - Titulação de ácido tetraprótl- CO $\left(\mathrm{H}_{4} \mathrm{~A}\right)$ COM UMA BASE FORTE (MOH)}

No caso tetraprótico a titulação processa-se em 4 passos:

$$
\begin{aligned}
& \mathrm{H}_{4} \mathrm{~A}(a q)+\mathrm{OH}^{-}(a q) \rightarrow \mathrm{H}_{3} \mathrm{~A}^{-}(a q)+\mathrm{H}_{2} \mathrm{O}(l) \\
& \mathrm{H}_{3} \mathrm{~A}^{-}(a q)+\mathrm{OH}^{-}(a q) \rightarrow \mathrm{H}_{2} \mathrm{~A}^{2-}(a q)+\mathrm{H}_{2} \mathrm{O}(l) \\
& \mathrm{H}_{2} \mathrm{~A}^{2-}(a q)+\mathrm{OH}^{-}(a q) \rightarrow \mathrm{HA}^{3-}(a q)+\mathrm{H}_{2} \mathrm{O}(l) \\
& \mathrm{HA}^{3-}(a q)+\mathrm{OH}^{-}(a q) \rightarrow \mathrm{A}^{4-}(a q)+\mathrm{H}_{2} \mathrm{O}(l)
\end{aligned}
$$

Em qualquer instante da titulação o equilíbrio iónico estabelecido na solução titulada é dado por:
$\left[\mathrm{H}^{+}\right]+\left[\mathrm{M}^{+}\right]=\left[\mathrm{OH}^{-}\right]+\left[\mathrm{H}_{3} \mathrm{~A}^{-}\right]+$

$+2\left[\mathrm{H}_{2} \mathrm{~A}^{2-}\right]+3\left[\mathrm{HA}^{3-}\right]+4\left[\mathrm{~A}^{4-}\right]$

sendo as concentrações do ácido tetraprótico ionizado dadas por:

$\left[\mathrm{H}_{3} \mathrm{~A}^{-}\right]+2\left[\mathrm{H}_{2} \mathrm{~A}^{2-}\right]+3\left[\mathrm{HA}^{3-}\right]+4\left[\mathrm{~A}^{4-}\right]=$

$=\frac{C_{b} \cdot V_{e q}}{V_{a}+V_{b}} \cdot\left(\alpha_{1}+2 \alpha_{2}+3 \alpha_{3}+4 \alpha_{4}\right)$

Por substituição de (3.2) em (3.1) obtém-se a expressão que após simplificação fica:

$\left[\mathrm{H}^{+}\right]-\frac{K_{w}}{\left[\mathrm{H}^{+}\right]}+\frac{C_{b}}{V_{a}+V_{b}}$.

. $\left(V_{b}-\alpha_{a 4}^{*} \cdot V_{e q}\right)=0$

onde

$$
\alpha_{a 4}^{*}=\sum_{i=1}^{4}\left(i . \alpha_{i}\right)=
$$

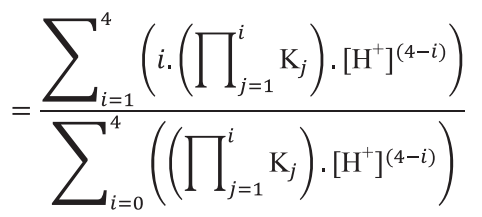

representa a fracção molar equivalente de ácido tetraprótico (a4) que se apresenta em solução aquosa sob a forma ionizada. $C_{\text {ASO }} 4$ - Titulação de ÁCIDO POLlPRótICO
$\left(\mathrm{H}_{\mathrm{n}} \mathrm{A}\right)$ COM UMA BASE FORTE (MOH)

Dos casos anteriores resulta que é relativamente fácil prever uma situação genérica para um ácido poliprótico que englobe também estes casos.

No caso poliprótico a titulação processa-se em $n$ passos:

$\mathrm{H}_{n} \mathrm{~A}(a q)+\mathrm{OH}^{-}(a q) \rightarrow \mathrm{H}_{(n-1)} \mathrm{A}^{-}(a q)+\mathrm{H}_{2} \mathrm{O}(l)$

$\mathrm{H}_{(n-1)} \mathrm{A}^{-}(a q)+\mathrm{OH}^{-}(a q) \rightarrow \mathrm{H}_{(n-2)} \mathrm{A}^{2-}(a q)+\mathrm{H}_{2} \mathrm{O}(l)$

$\mathrm{HA}^{(n-1)-}(a q)+\mathrm{OH}^{-}(a q) \rightarrow \mathrm{A}^{n-}(a q)+\mathrm{H}_{2} \mathrm{O}(l)$

De modo similar, ao longo da titulação estabelece-se um equilíbrio iónico na solução de titulado que é dado por:
$\left[\mathrm{H}^{+}\right]+\left[\mathrm{M}^{+}\right]=\left[\mathrm{OH}^{-}\right]+\left[\mathrm{H}_{(n-1)} \mathrm{A}^{-}\right]+$

$+2\left[\mathrm{H}_{(n-2)} \mathrm{A}^{2-}\right]+\ldots+1$

$+(n-1)\left[\mathrm{HA}^{(n-1)-}\right]+n\left[\mathrm{~A}^{n-}\right]$

As concentrações do ácido que se encontram sob a forma ionizada são dadas por:

$$
\begin{aligned}
& {\left[\mathrm{H}_{(n-1)} \mathrm{A}^{-}\right]+2\left[\mathrm{H}_{(n-2)} \mathrm{A}^{2-}\right]+\ldots+n\left[\mathrm{~A}^{n-}\right]=} \\
& =\frac{C_{b} \cdot V_{e q}}{V_{a}+V_{b}} \cdot\left(\alpha_{1}+2 \alpha_{2}+\ldots+n \alpha_{n}\right)
\end{aligned}
$$

Por substituição de (4.2) em (4.1) e após alguma simplificação obtém-se o modelo que descreve a curva de titulação:

$$
\begin{aligned}
& {\left[\mathrm{H}^{+}\right]-\frac{K_{w}}{\left[\mathrm{H}^{+}\right]}+\frac{C_{b}}{V_{a}+V_{b}} .} \\
& .\left(V_{b}-\alpha_{a n^{*}}^{*} V_{e q}\right)=0
\end{aligned}
$$

onde

$$
\begin{aligned}
& \alpha_{a n}^{*}=\sum_{i=1}^{n}\left(i . \alpha_{i}\right)= \\
& =\frac{\sum_{i=1}^{n}\left(i \cdot\left(\prod_{j=1}^{i} \mathrm{~K}_{j}\right) \cdot\left[\mathrm{H}^{+}\right]^{(n-i)}\right)}{\sum_{i=0}^{n}\left(\left(\prod_{j=1}^{i} \mathrm{~K}_{j}\right) \cdot\left[\mathrm{H}^{+}\right]^{(n-i)}\right)}
\end{aligned}
$$

representa a fracção molar equivalente de ácido poliprótico (an) que se apresenta em solução sob a forma ionizada.

\section{GeneralizaÇÃo do MOdelo e MOdO DE RESOLUÇÃO}

Comparando as equações dos modelos para os quatro casos distintos aqui deduzidos e, confrontando ainda com os resultados anteriormente deduzidos [6], salienta-se a existência de um modelo genérico que descreve a curva de titulação de uma espécie ácida (poliprótica ou não) através de:

$$
\left[\mathrm{H}^{+}\right]-\frac{K_{w}}{\left[\mathrm{H}^{+}\right]}+\varphi^{*}=0
$$

onde a concentração da fracção titulada $\left(\varphi^{*}\right)$ assume a forma: 


$$
\varphi^{*}=\frac{C_{b} \cdot\left(V_{b}-\alpha_{a n^{*}}^{*} V_{e q}\right)}{V_{a}+V_{b}}
$$

e em que $\alpha_{a n}^{*}$ representa a fracção molar equivalente de ácido que se apresenta em solução sob a forma ionizada, equação (4.4).

Como foi anteriormente salientado, a previsão da curva de titulação não pode ser obtida por via directa, através de uma resolução analítica.

De uma forma sucinta, o algoritmo numérico proposto [6] baseia-se na resolução aproximativa das equações (1.3), (2.3), (3.3) e (4.3) por via iterativa: a) inicialmente assume-se um determinado valor de $\mathrm{pH}$ em cada ponto da curva de titulação, b) calcula-se a concentração hidrogeniónica em cada instante da titulação, equação (0.5), c) estima-se a respectiva fracção titulada, equação (5.2), d) calcula-se o valor previsto pelo modelo, equação (5.1) e e) estima-se o erro do modelo sob a forma de soma de quadrados (SS):

$$
\mathrm{SS}=\sum_{i=1}^{N}\left(e_{i}{ }^{2}\right)=\sum_{i=1}^{N}\left(\left[\mathrm{H}^{+}\right]-\frac{K_{w}}{\left[\mathrm{H}^{+}\right]}+\varphi^{*}\right)^{2}
$$

As iterações são conduzidas por alteração do valor de $\mathrm{pH}$ e no sentido de minimizar o erro previsto pelo modelo, equação (5.3).

Como foi previamente demonstrado [6], a estimativa de curvas de titulação pode ser facilmente implementada numa folha de cálculo com o Excel, não sendo para tal necessário avançados conhecimentos em programação.

Este tipo de folha de cálculo permite, entre outras vantagens, utilizar uma poderosa ferramenta de optimização (suplemento SOLVER) e obter em simultâneo uma aceitável representação gráfica, automaticamente actualizada após cada cálculo. Esta "macro" do Excel recorre ao algoritmo GRG2 ${ }^{5}$ que foi desenvolvido especialmente para lidar com problemas de optimização envolvendo funções não lineares complexas [7, 8].

\section{Aplicações}

Considerando sempre a mesma base forte $(\mathrm{NaOH} 0.200 \mathrm{M})$ como titulante, foram estimadas as curvas de titulação de $20.0 \mathrm{~mL}$ de solução aquosa contendo um ácido $\left(\mathrm{C}_{\mathrm{a}}=0.100 \mathrm{M}\right)$ a) diprótico $\left(\mathrm{H}_{2} \mathrm{~A}\right.$, exemplos dos ácidos p-hidroxibenzóico e o-ftálico), $b$ ) triprótico $\left(\mathrm{H}_{3} \mathrm{~A}\right.$, exemplos dos ácidos fosfórico e cítrico), c) tetraprótico $\left(\mathrm{H}_{4} \mathrm{~A}\right.$, exemplo do ácido pirofosfórico) e hexaprótico $\left(\mathrm{H}_{6} \mathrm{~A}\right.$, EDTA). Como será mais à frente esclarecido, as espécies ácidas seleccionadas para estudo apresentam valores de constantes de equilíbrio criteriosamente escolhidas [9] de forma a poder extrair mais ilações relacionadas com a forma da curva de titulação.

Nas condições escolhidas, em todas as titulações os pontos de equivalência vão surgir para volumes de titulante múltiplos de $10.0 \mathrm{~mL}$, equação (0.6).

$\mathrm{Na}$ figura 1 encontram-se representadas duas curvas de titulação de um ácido diprótico (caso 1) com base forte, em duas situações distintas, atendendo aos respectivos valores das constantes de equilíbrio escolhidas.

Da figura 1 verifica-se que a forma das curvas é muito distinta - enquanto que no primeiro caso as constantes diferem mais de 4 ordens de grandeza, sendo possível distinguir as duas fases distintas da curva de titulação, separadas pelos pontos de equivalência $\left(V_{b}=10.0\right.$ e $\left.20.0 \mathrm{~mL}\right)$, no segundo caso as constantes são mais próximas (diferem cerca de uma ordem de

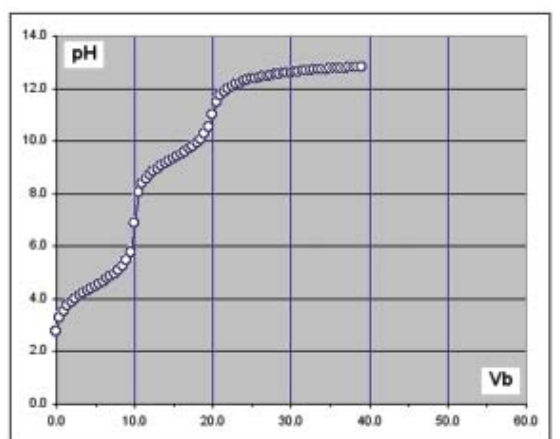

(A) - ácido para-hidroxibenzóico [pK's $=4.48,9.32$ ] grandeza) não sendo possível identificar o primeiro ponto de equivalência (10.0 mL).

Neste último caso a curva de titulação assemelha-se à de um ácido monoprótico com o dobro de concentração.

Na figura 2 apresentam-se dois casos de titulações com ácidos tripróticos (caso 2) com comportamentos distintos.

No primeiro caso as constantes sucessivas diferem cerca de cinco ordens de grandeza sendo possível evidenciar os dois primeiros pontos de equivalência $\left(V_{b}=10.0\right.$ e $\left.20.0 \mathrm{~mL}\right)$. O terceiro ponto de equivalência (30.0 $\mathrm{mL}$ ) não é perceptível dado que a base forte utilizada como titulante não é muito concentrada $(0.200 \mathrm{M})$, limitando as medições ao valor máximo de $\mathrm{pH}$ inferior a 13. No segundo caso as constantes de equilíbrio diferem cerca de uma ordem de grandeza não sendo evidenciados os dois primeiros pontos de equivalência $\left(\mathrm{V}_{\mathrm{b}}=10.0 \mathrm{e}\right.$ $20.0 \mathrm{~mL}$ ) mas apenas o terceiro ponto de equivalência $(30.0 \mathrm{~mL})$ - este tipo de comportamento assemelha-se ao de um ácido monoprótico três vezes mais concentrado.

Na figura 3 encontram-se as curvas de titulação para os casos de ácido tetraprótico e hexaprótico.

Da figura 3 verifica-se uma vez mais que apenas são perceptíveis os pontos de inflexão quando as constantes de acidez sucessivas diferem entre si cerca de três ordens de grandeza.

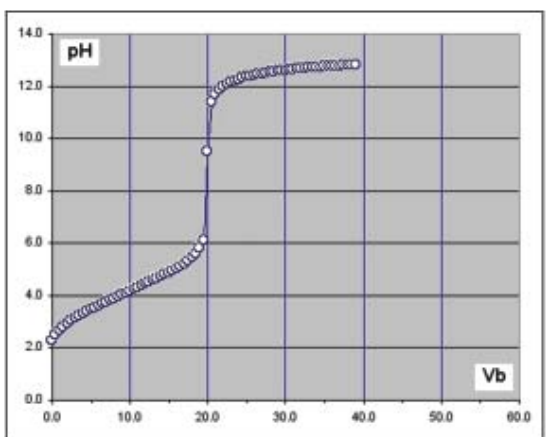

(B) - ácido orto-ftálico [pK's = 3.51, 4.82]

Figura 1 - Curvas de titulação previstas para a reacção de um ácido diprótico com uma base forte (MOH): casos do (A) ácido p-hidroxibenzóico e (B) ácido o-ftálico (indicação do valor das constantes utilizadas em cada caso na forma logaritmizada, pK) 
No sentido de verificar qual a diferença mínima na ordem de grandeza das sucessivas constantes de equilíbrio que permite evidenciar uma inflexão na curva de titulação, foi realizada uma simulação considerando um ácido tetraprótico. Fixou-se o valor para a primeira constante de acidez $\left(\mathrm{pK}_{1}=\right.$ 0.00 ) e, em cada caso, considerou-se sempre um intervalo idêntico para a diferença de ordem de grandeza entre constantes sucessivas $\left(\Delta \mathrm{pK}=\mathrm{pK}_{\mathrm{i}}\right.$ - $\left.\mathrm{pK}_{(\mathrm{i}-1)}\right)$. Foram testadas amplitudes $(\Delta \mathrm{pK})$ a variar entre 1.0 e $4.0 \mathrm{com}$ incrementos de 0.5. No primeiro caso simulado $(\Delta \mathrm{pK}=1)$ as constantes utilizadas são pK's $=0.00,1.00,2.00$ e 3.00 , enquanto que no último caso $(\Delta \mathrm{pK}=4)$ as constantes utilizadas são pK's $=0.00,4.00,8.00$ e 12.00 .

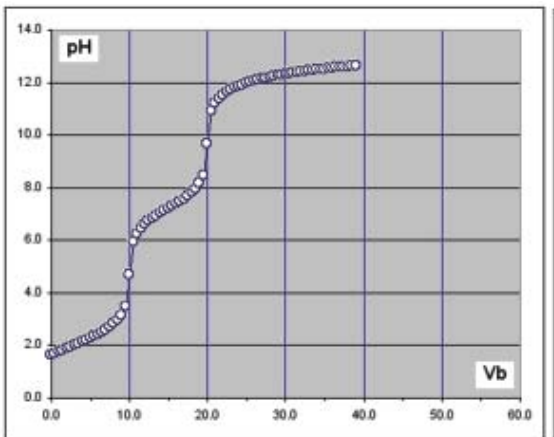

(A) - ácido fosfórico [pK's $=2.15,7.20,12.35]$

do titulado é composto por grupos ácidos com carácter distinto. Contudo, a inflexão na curva de titulação apenas se torna perfeitamente nítida a partir de uma diferença de três ordens de grandeza nas respectivas constantes.

Através desta última figura é ainda possível retirar outra conclusão: a manifestação do comportamento ácido das espécies presentes no titulado está confinada a uma faixa de $\mathrm{pH}$ (situada entre o valor inicial e final da curva de titulação), imposta pelas condições experimentais, que suprime a manifestação do carácter ácido das espécies com constantes de ionização situadas fora desta janela experimental utilizada no estudo deste fenómeno.

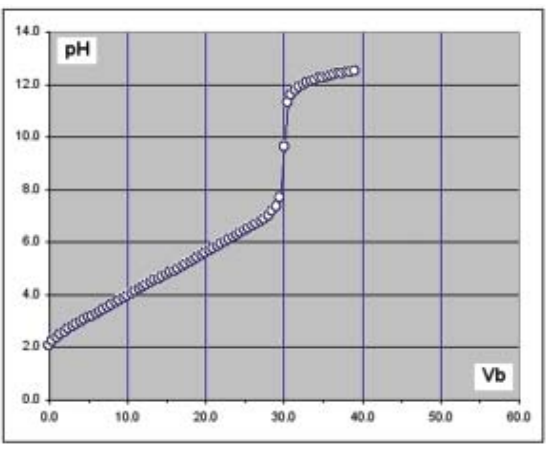

(B) - ácido cítrico $[\mathrm{pK}$ 's $=3.13,4.76,6.40]$

Figura 2 - Curvas de titulação previstas para a reaç̧ão de um ácido triprótico com base forte $(\mathrm{MOH})$ : casos do (A) ácido fosfórico e (B) ácido cítrico (indicação do valor das constantes utilizadas em cada caso)

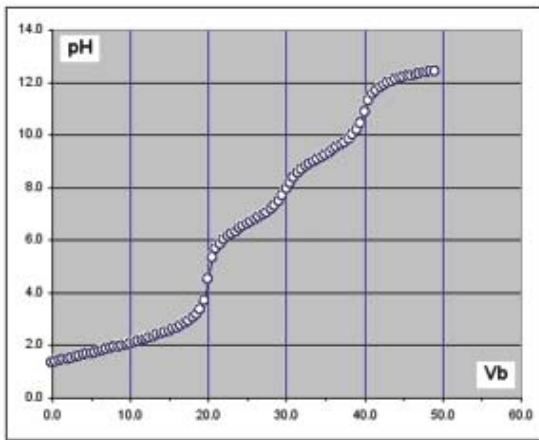

(A) - ácido pirofosfórico [pK's $=1.52,2.36,6.60,9.25]$

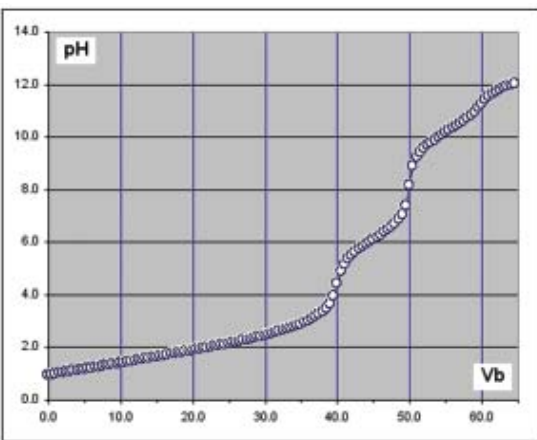

(B) - EDTA ${ }^{6}$

$\left[\mathrm{pK}^{\prime} \mathrm{s}=0.00,1.50,2.00,2.68,6.11,10.17\right]$

Figura 3 - Curvas de titulação previstas para um ácido (A) tetraprótico e (B) hexaprótico

Os resultados obtidos encontram-se representados na figura 4.

Através da figura 4 pode-se verificar que, com apenas duas ordens de grandeza na diferença sucessiva do valor das constantes $(\Delta \mathrm{pK})$, já é possível evidenciar que o sistema químico
Foi ainda verificado que a expressão genérica deduzida para o modelo da curva de titulação de um ácido poliprótico com uma base forte $(\mathrm{MOH})$, equação (5.1), é não só válida para qualquer caso de titulação de um ácido mono/poliprótico fraco como ainda para o caso de ácido forte (HX).

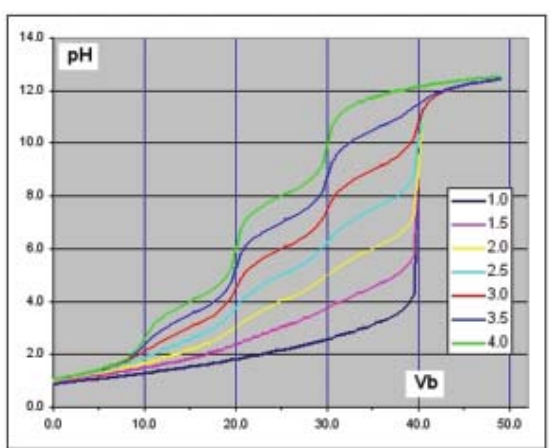

Figura 4 - Simulação de curvas de titulação para o caso de um ácido tetraprótico $\left[\mathrm{pK}_{1}=0.00, \mathrm{pK}_{\mathrm{i}}=\mathrm{i}\right.$. $\Delta \mathrm{pK}]$. Na legenda da figura encontra-se indicada a amplitude utilizada na diferença sucessiva das constantes de ionização, medida em ordens de grandeza $(\Delta \mathrm{pK})$

Salientamos também que este processo de cálculo não apresenta ainda qualquer dificuldade numérica adicional ao tratar sistemas polipróticos com equilíbrios sequenciais (constantes sucessivas com valores muito distintos) ou simultâneos (constantes com valores mais próximos), o que revela a enorme vantagem do modelo e abordagem matemática aqui descrita.

\section{NotAS:}

Esta ferramenta de cálculo pode ser obtida mediante solicitação dos interessados por via e-mail para jcpereira@qui.uc.pt sob o assunto "Previsão de curvas de titulação: pedido de envio da folha de cálculo PrevCTPPoli. xls".

1 De acordo com um trabalho publicado por Norris F. Hall em 1940 ("Systems of Acids and Bases", J. Chem. Educ. 17(1940)124) a teoria de reactividade do tipo ácido-base conhecida por BrønstedLowry foi proposta simultaneamente por Johannes Nicolaus Brønsted (Dinamarquês) e por Thomas Martin Lowry (Inglês) em 1923 tendo estes autores desenvolvido os seus conceitos de uma forma autónoma e independente.

2 Uma espécie química diz-se anfifílica se puder comportar-se como ácido ou como base, conforme as condições do meio em que esta se encontra.

${ }^{3}$ Uma espécie monobásica é uma base que reage recebendo apenas um protão.

${ }^{4}$ Apenas válido se fossem mantidas constantes a temperatura, a pressão e a força iónica do meio.

${ }^{5}$ A sigla GRG2 significa Generalized Reduced Gradient, que é um método numérico especialmente concebido para pesquisa de soluções, orientando a sua 
busca através do cálculo dos respectivos gradientes da função de mérito.

${ }^{6}$ EDTA, ácido etilenodiamina tetraacético.

\section{REFERÊNCIAS}

[1] R. Chang, Chemistry, $8^{a}$ Ed., McGrawHill, New York, 2004.

[2] P. Atkins and L. Jones, Chemical Principles: the quest for insight, $3^{\text {th }} \mathrm{Ed}$.,
Freeman, New York, 2005.

[3] D.L. Nelson, M.M. Cox, Lehninger Principles of Biochemistry, $5^{\text {th }}$ Ed., Freeman, London, 2008.

[4] G.K. Hunter, Vital Forces: The Discovery of the Molecular Basis of Life, Academic Press, San Diego, 2000.

[5] R.O. James, J.A. Davis, J.O. Leckie, J. Colloid Interface Sci., 65 (1978) 331.

[6] J.L.G.F.S.C. Pereira, Previsão de curvas de titulação: uma abordagem quantitativa, Química, 112 (2009) 19.

[7] R. Maran, Microsoft Excel Simplified, Wiley, New York, 1999.

[8] E.J. Billo, Excel for Scientists and Engineers Numerical Methods, Wiley, New York, 2007.

[9] D.R. Lide, CRC Handbook of Chemistry and Physics, 87th Ed., CRC Press, London, 2006.

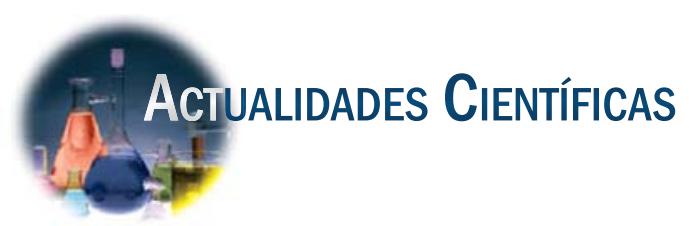

\section{TAMANHO de CARBENos Controla ACoplamentos}

Recorrendo a ligantes de carbeno, uma equipa de químicos descobriu novas formas de controlar reacções de acoplamento para síntese de álcoois alílicos.

A escolha entre ligantes de um catalisador de níquel de pequena ou elevada dimensão determina qual a região de um alcino que acoplará a um aldeído para a formação de um álcool, possibilitando um nível de controlo sobre a reacção que os químicos de síntese têm até ao momento revelado grandes dificuldades em atingir.

Os álcoois alílicos são comuns no campo da produção de antibióticos e de outros produtos farmacêuticos. Apesar de já terem sido conseguidos anteriormente processos de acoplamento catalítico e enantioselectivo entre aldeídos e alcinos, o controlo da regioquímica destas reacções através de catalisadores metálicos tem-se revelado problemático.

Para evitar a obtenção de misturas não controladas de produtos reac- cionais, os investigadores focaram a sua atenção nas propriedades de alcinos que denotem uma alta tendência para um determinado tipo de isómero estrutural ou que possuam grupos funcionais que influenciem a selectividade da reacção. Estas questões limitam consideravelmente a utilidade da reacção.

No entanto, John Montgomery e os alunos de graduação Hasnain A. Malik e Grant J. Sormunen da Universidade de Michigan, em Ann Arbor, verificaram que o tamanho dos ligantes de carbeno N-heterocíclicos do catalisador de níquel dita o resultado regioquímico das reacções (J. Am. Chem. Soc., DOI: 10.1021/ ja102262v). Ligantes maiores levam à formação de ligações na extremidade menos acessível do alcino, enquanto que ligantes pequenos conduzem ao resultado contrário. De momento, nenhum ligante específico proporciona um cenário óptimo.

Através do seu método a equipa conseguiu controlar a regioselectivida- de de alcinos menos tendenciosos e contrariar a expectável selectividade de alcinos notoriamente direccionados. Agora, a equipa pretende estudar uma gama mais alargada de alcinos e de aldeídos de forma a testar a generalidade do fenómeno, para além de potenciar o desempenho dos ligantes.

O trabalho do grupo Montgomery "resolve um problema já antigo dentro do campo da reactividade química e proporciona um exemplo paradigmático do impacto que os diversos carbenos $\mathrm{N}$-heterocíclicos podem assumir no controlo das reacções catalíticas", afirma o professor de Química Glenn Micalizio do Scripps Research Institute, Florida, cujo laboratório desenvolve igualmente reacções de acoplamento regioselectivas.

(Adaptado do artigo de 23/04/2010 de Carmen Drahl: Carbene Size

Guides Coupling Chemical \& Engineering News - http:// pubs.acs.org/ cen/news/88/i17/8817notw7.html)

PB

\section{DigA-NOS O QUE GOSTARIA DE VER NO "QUÍMICA"}

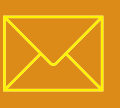

ENVIE-NOS A SUA OPINIÃO E SUGESTÕES PARA bquimica@ipb.pt 
